\title{
Glutathione S-Transferases and Cytochrome P450 Enzyme Expression in Patients with Intracranial Tumors: Preliminary Report of 55 Patients
}

\author{
Cahit Kural $^{a}$ Arzu Kaya Kocdogan ${ }^{b}$ Gulcin Güler Şimşek ${ }^{c}$ Serpil Oğuztüzün ${ }^{b}$ \\ Pınar Kaygın ${ }^{b}$ Irmak Yılmaz ${ }^{b}$ Tugbag Bayram $^{b}$ Yusuf Izcia \\ ${ }^{a}$ Department of Neurosurgery, University of Health Sciences and Gulhane Education and Research Hospital, \\ Ankara, Turkey; ${ }^{\text {b }}$ Department of Biology, Kırıkkale University, Kırıkkale, Turkey; ${ }^{c}$ Department of Pathology, University \\ of Health Sciences and Kecioren Education and Research Hospital, Ankara, Turkey
}

\section{Significance of the Study}

- In this study, it was found that GSTP1 and CYP expression is increased in intracranial tumors. In addition, pituitary adenomas may show the highest GST and CYP expression. These tumors may therefore have higher drug-metabolizing capacities than other intracranial tumors.

\section{Keywords}

Brain · Tumor biology · Glutathione-S-transferase •

Cytochrome p450

\begin{abstract}
Objective: Intracranial tumors are one of the most frightening and difficult-to-treat tumor types. In addition to surgery, protocols such as chemotherapy and radiotherapy also take place in the treatment. Glutathione S-transferase (GST) and cytochrome P450 (CYP) enzymes are prominent drug-metabolizing enzymes in the human body. The aim of this study is to show the expression of GSTP1, GSTM1, CYP1A1, and CY$\mathrm{P} 1 \mathrm{~B} 1$ in different types of brain tumors and compare our results with those in the literature. Subjects and Methods: The expression of GSTP1, GSTM1, CYP1A1, and CYP1B1 was analyzed using immunostaining in 55 patients with intracranial
\end{abstract}

\begin{tabular}{ll}
\hline KARGER & $\begin{array}{l}\text { (c) 2018 The Author(s) } \\
\text { Published by S. Karger AG, Basel }\end{array}$ \\
E-Mail karger@karger.com & $\begin{array}{l}\text { This is an Open Access article licensed under the Creative Commons } \\
\text { Attribution-NonCommercial-4.0 International License (CC BY-NC) } \\
\text { (http://www.karger.com/Services/OpenAccessLicense), applicable to } \\
\text { the online version of the article only. Usage and distribution for } \\
\text { commercial purposes requires written permission. }\end{array}$
\end{tabular}

tumors in 2016-2017. For GST and CYP expression in normal brain tissue, samples of a portion of surrounding normal brain tissue as well as a matched far neighbor of tumor tissue were used. The demographic features of the patients were documented and the expression results compared. Results: The mean age of the patients was 46.72 years; 29 patients were female and 26 were male. Fifty-seven specimens were obtained from 55 patients. Among them, meningioma was diagnosed in 12, metastases in 12, glioblastoma in 9, and pituitary adenoma in 5 . The highest GSTP1, GSTM1, and CYP$1 \mathrm{~A} 1$ expressions were observed in pituitary adenomas. The lowest GSTP1 expression was detected in glioblastomas and the lowest CYP1B1 expression in pituitary adenomas. Conclusion: GSTP1 and CYP expression is increased in intracranial tumors. These results should be confirmed with a larger series and different enzyme subtypes.

(C) 2018 The Author(s)

Published by S. Karger AG, Basel 


\section{Introduction}

Intracranial tumors may involve the brain or other related structures, such as the meninges, pituitary gland, pineal gland, and cranial nerves [1]. Intracranial tumors can also be found in about $2 \%$ of routine autopsies [2]. Some intracranial tumors are benign, but because the cranial vault has no additional space for expansion, malignant and even benign tumors may cause significant neurological deficits or death due to increased intracranial pressure [3].

The histopathological features of intracranial tumors influence their clinical presentation and the prognosis of diseases [4]. Intracranial tumors are of 2 types: primary tumors that originate in the brain, either in the brain parenchyma or in extraneural structures, and secondary tumors that originate in tissues outside the brain (such as the lung, breast, and kidney), and spread into the intracranial space. Intracranial metastases are more common than primary brain tumors and also the most common malignant intracranial tumors along with glial tumors [5].

Chemoresistance is one of the important problems in the treatment of intracranial tumors. Several mechanisms of drug resistance have been proposed [6]. Intracellular drug inactivation or metabolism may occur as a result of increased concentrations of enzymes such as glutathione S-transferase (GST) or cytochrome p450 (CYP). These enzymes may play a role in reducing the efficacy of chemotherapy against malignant tumors $[7,8]$. Glioblastomas (GBMs) are astrocytic tumors and account for about $50 \%$ of all gliomas [9]. They exhibit extremely high local invasiveness. Alkylating agents are becoming increasingly used for the systemic chemotherapy of GBMs $[9,10]$. One of the major problems in the chemotherapy of GBM is chemoresistance [11]. The sensitivity of astrocytes to alkylating agents is significantly increased by blocking the DNA-repair enzyme MGMT and by blocking glutathione synthesis [11].

GST genes are upregulated in response to oxidative stress and are inexplicably overexpressed in many tumors, leading to problems during cancer chemotherapy. There are many studies regarding the relationship between GST genotypes and cancer of the breast, lung, colon, brain, bladder, prostate, and other organs [12].

Human cytosolic GSTs have been grouped into several families according to sequence homology and immunological cross-reactivity. The most frequently mentioned are designated as GST $\mu$ (GSTM1), GST $\theta$ (GSTT1), and GST $\pi$ (GSTP1) $[12,13]$. In the brain, GSTs are located primarily in astrocytes, possibly playing a neuro-

Glutathione-S-transferase and

Cytochrome p450 in Brain Tumors protective role. On the other hand, elevated expression of GSTs may have an impact on the chemotherapeutic effect. This effect is either by direct drug metabolism or by potentially reducing the ability of the drugs to interact with DNA and other cellular molecules [13].

CYPs are the most important phase I drug metabolizing enzymes, adding an oxygen atom to their substrate [14]. The enzymes studied are of particular interest since they seem to have an important extrahepatic function and have been linked to various malignancies. Cytochrome p450 1A1 (CYP1A1) is the enzyme responsible for the aryl-hydrocarbon hydroxylase activity. It is involved in the metabolic activation of several carcinogenic substances $[8,15]$. A role of CYP1A1 in lung cancer risk was already proposed 30 years ago $[15,16]$. The initial hypothesis of the occurrence of an inducibility polymorphism, together with the role of CYP1A1 in the metabolic activation of tobacco carcinogens, provided support for several studies on smoking-related cancers and CYP1A1 polymorphisms. However, further studies failed to confirm a clear association between polymorphisms of the CYP1A1 gene and enzyme inducibility $[15,16]$. Available information on other types of cancer is limited, either because in the studies published involve small sample sizes, or are single reports without independent confirmation by other research groups [15]. Cytochrome p450 1B1 (CYP1B1) catalyzes the 4-hydroxylation of estrogens, a reaction of key relevance in hormonal carcinogenesis. In addition, the enzyme activates many environmental mutagens [16]. CYP1B1 is overexpressed in diverse types of cancer including that of the lungs, breasts, liver, gastrointestinal tract, ovaries, prostate, and bladder. Interestingly, the protein is absent or expressed to a minor extent in healthy tissues $[16,17]$.

In order to discover potential markers to identify patients with intracranial tumors who are at risk of chemotherapy failure and adverse effects, we conducted a preliminary study on 57 tumor specimens in 55 patients. Predictive biomarkers in brain tumors can indicate the likely effect of specific adjuvant therapies on patient outcomes such as tumor recurrence or survival. GSTs detoxify chemotherapeutic drugs by catalyzing the reduction of these compounds through their conjugation with glutathione. CYPs constitute the major enzyme family capable of catalyzing the oxidative biotransformation of most chemotherapeutic agents. For this reason, we focused on these enzymes, and the expression of GSTP1, GSTM1, CYP$1 \mathrm{~A} 1$, and CYP1B1 in different brain tumors was analyzed. Possible correlations between the levels of expression and types of brain tumor were investigated.

Med Princ Pract 2019;28:56-62

DOI: $10.1159 / 000494496$ 


\section{Material and Methods}

Information on patients undergoing surgery for intracranial tumors and performed by members of the Gulhane Education and Research Hospital Department of Neurosurgery was entered into a prospective computer database between June 2016 and June 2017. The data were evaluated at the end of June 2017.

The dataset contains information on patient demographics, histopathology, and enzyme expressions. All patients gave written consent personally or through their guardian for pathology specimens and anonymous clinical data to be used for research purposes. All resections were performed by neurosurgeons according to a standardized procedure, and the acquisition of clinical data was made by a neurosurgeon. Patients reported here had a resection for an intracranial tumor between 2016 and 2017.

A total of 55 patients underwent surgical removal of a brain tumor in a 6-month period and 57 brain tumors were analyzed. Two patients had 2 tumors each, at different locations. Three of 57 specimens were radiation necrosis and 54 were tumor samples. GSTP1 and GSTM1, CYP1A1, and CYP1B1 were analyzed in the tumor tissues of the patients. In addition, these markers were also analyzed in normal brain-tissue specimens obtained from the surrounding tissue. The expression of enzymes was classified as 0,1 , 2 , or 3 based on microscopic examination after immunostaining.

\section{Histopathological Examination}

Pathological examination of the resected specimens followed a standard protocol as described previously. Surgical specimens were examined macroscopically by a pathologist in each case and 2 tissue samples were obtained from these specimens: 1 from the tumor tissue and 1 from the macroscopically normal tissue peripheral to the tumor. Only intracranial tumors (including gliomas, metastases, meningiomas, and pituitary adenomas) were included in the dataset. Where multiple tumors were present, all were removed and examined.

Tumor size was measured as the greatest surface dimension. Blocks were taken to demonstrate maximum direct tumor penetration of the brain. Additional blocks were taken specifically to demonstrate the relationship between the tumor and any adherent structure or tissue as well as the lines of resection and the normal brain tissue. Tumor grade was assessed by taking into account the degree of differentiation and anaplasia, the nature of the tumor margin (pushing or infiltrating), and the presence and prominence of brain invasion. All pathological characteristics analyzed were looked for in every specimen, and their presence or absence was recorded in detail. For GST and CYP expression in normal brain tissue, samples of a portion of surrounding normal brain tissue as well as a matched far neighbor of tumor tissue were used. Expressions of follicle-stimulating hormone (FSH), luteinizing hormone $(\mathrm{LH})$, prolactin $(\mathrm{PRL})$, growth hormone $(\mathrm{GH})$, and adrenocorticotropic hormone (ACTH) were analyzed in pituitary adenoma samples. Ki-67 expression was also assessed for each tumor by immunohistochemical techniques.

\section{Immunohistochemical Staining}

Tissues were fixed in $10 \%$ buffered formalin and embedded in paraffin blocks. Sections that were $4 \mu \mathrm{m}$ thick were cut, and one section was stained with hematoxylin \& eosin (HE) to observe the tissue morphology. For immunohistochemistry, endogenous peroxidase activity was blocked by incubating the sections in $1 \%$ hy-
Table 1. Distributions of the tumor samples according to histological diagnosis

\begin{tabular}{lrr}
\hline Tumor (lesion) type & $n$ & $\%$ \\
\hline Meningioma & 9 & 15.8 \\
$\quad$ Grade I & 3 & 5.2 \\
Grade II & 12 & 21.1 \\
Getastasis & 9 & 15.8 \\
Plioblastoma & 5 & 8.8 \\
Anaplastic astrocytoma & 3 & 5.2 \\
Anaplastic oligodendroglioma & 2 & 3.5 \\
Oligodendroglioma & 2 & 3.5 \\
Pilocytic astrocytoma & 2 & 3.5 \\
Central neurocytoma & 2 & 3.5 \\
Gliosarcoma & 2 & 3.5 \\
Schwannoma & 1 & 1.8 \\
Medulloblastoma & 1 & 1.8 \\
Lhermitte-Duclos disease & 1 & 1.8 \\
Radiation necrosis & 3 & 5.2 \\
\hline Total & $\mathbf{5 7}$ & $\mathbf{1 0 0 . 0}$ \\
\hline
\end{tabular}

drogen peroxide $(\mathrm{v} / \mathrm{v})$ in methanol for $10 \mathrm{~min}$ at room temperature (RT). The sections were subsequently washed in distilled water for $5 \mathrm{~min}$, and antigen retrieval was performed for $3 \mathrm{~min}$ using $0.01 \mathrm{M}$ citrate buffer ( $\mathrm{pH}$ 6.0) in a domestic pressure cooker. After washing in distilled water, the sections were transferred in $0.05 \mathrm{M}$ Tris$\mathrm{HCl}$ ( $\mathrm{pH} 7.6)$ containing $0.15 \mathrm{M}$ sodium chloride (TBS). The sections were incubated at RT for 10 min with Super Block (streptavidin/HRP complex [SHP125]; ScyTek Laboratories, USA) to block nonspecific background staining. The sections were then covered with the primary antibodies diluted 1:500 for anti-GSTP1, 1:1,000 for anti-GSTM1, 1:50 for anti-CYP1A1, 1:50 for anti-CYP$1 \mathrm{~B} 1$ in TBS at $4^{\circ} \mathrm{C}$ overnight (anti-GSTP1 [LS-C211876] from Boster Biological, Pleasanton, CA, USA; anti-GSTM [NBP222186] from Novus Biologicals, Littleton, CO, USA; anti-CYP1A1 [sc-20772] and anti-CYP1B1 [sc-32882] were from Santa Cruz Biotechnology Inc., USA). After washing in TBS for $15 \mathrm{~min}$, the sections were incubated at RT for biotinylated link antibody followed with SHP125. Diaminobenzidine was used to visualize peroxidase activity in the tissues. Nuclei were lightly counterstained with hematoxylin, and then the sections were dehydrated and mounted. Both positive and negative controls were included in each run.

Light microscopy of immunohistochemically stained sections was performed by 2 pathologists who were unaware of the patients' clinical information, other histopathological data, or survival. Tissue cores from the central part of the tumor and the invasive front were assessed separately in each sample, as was the presence of nuclear and cytoplasmic staining in the tumor epithelial cells. The intensity of staining was graded as 0 (no staining), 1 (weak staining), 2 (moderate expression), or 3 (strong staining).

\section{Follow-Up}

Apart from patients lost to follow-up, all patients were followed annually until death, or for up to 1 year, or until July 2017.
58

Med Princ Pract 2019;28:56-62

DOI: $10.1159 / 000494496$
Kural/Kaya Kocdogan/Şimşek/

Oğuztüzün/Kaygın/Yılmaz/Bayram/Izci 
Table 2. Mean ( \pm SD) GST and CYP scores of patients with glioblastoma, metastasis, meningioma, and pituitary adenoma

\begin{tabular}{|c|c|c|c|c|c|}
\hline & \multicolumn{2}{|l|}{ GST } & \multicolumn{2}{|l|}{ CYP } & \multirow[t]{2}{*}{$p^{\mathrm{a}}$} \\
\hline & GSTP1 & GSTM1 & CYP1A1 & CYP1B1 & \\
\hline Glioblastoma $(n=9)$ & $1.00 \pm 0.75$ & $0.62 \pm 0.74$ & $1.37 \pm 0.51$ & $1.37 \pm 0.51$ & 0.274 \\
\hline Pituitary adenoma $(n=5)$ & $2.20 \pm 0.44$ & $0.80 \pm 0.44$ & $2.00 \pm .70$ & $0.80 \pm 0.83$ & $>0.05$ \\
\hline Meningioma $(n=12)$ & $1.30 \pm 0.75$ & $0.50 \pm 0.51$ & $1.80 \pm 1.07$ & $1.60 \pm 0.67$ & $>0.05$ \\
\hline Metastasis $(n=12)$ & $1.36 \pm 0.92$ & $0.08 \pm 0.28$ & $1.33 \pm 0.65$ & $1.20 \pm 0.83$ & 0.308 \\
\hline Normal tissue & $0.15 \pm 0.20$ & $0.17 \pm 0.20$ & $0.35 \pm 0.17$ & $0.23 \pm 0.17$ & 0.080 \\
\hline$p^{\mathrm{b}}$ & \multicolumn{4}{|c|}{0.052} & \\
\hline
\end{tabular}

First, all enzyme subgroups (GSTP1, GSTM1, CYP1A1, and CYP1B1) were evaluated statistically according to each tumor type and examined for statistical significance. $p$ value was calculated based on this evaluation. Only GST and CYP enzyme values were then evaluated and assessed statistically by Kruskal-Wallis test.

${ }^{\text {a }}$ Friedman test; ${ }^{\mathrm{b}}$ Kruskal Wallis test.

\section{Statistical Analysis}

The Kruskal-Wallis test was used to analyze the relationship between gender and the expression of GSTP1, GSTM1, CYP1A1, and CYP1B1. The Pearson correlation test was used to analyze the relationship between age and the expression of GSTP1, GSTM1, CYP1A1, and CYP1B1. The Friedman test is a method of assessing the difference between different measurements applied on the same sample. This test, which is the most statistically significant method, was used to measure the differences between the different enzymes used for each type of tumor. The level for two-tailed statistical significance was $p<0.05$. Analyses were performed with SPSS v15.0 for Windows (SPSS Inc., Chicago, IL, USA).

\section{Results}

Twenty-nine (52.7\%) patients were female and 26 were male. The mean age was 46.72 years (6-77 years) in the whole series. The mean age was 45.8 years for female patients and 47.7 years for male patients. Among the 26 male patients, 6 were smokers $(23.1 \%)$. In the female group, $4 / 29$ patients $(13.8 \%)$ were smokers. The most common histopathological diagnoses were: meningioma in $12(21 \%)$ samples, metastasis in $12(21.1 \%)$, and GBM in $9(15.8 \%)$. Radiation necrosis was diagnosed in 3 cases (Table 1).

There was no difference in expressions of GSTP1, GSTM1, CYP1A1, and CYP1B1 based on the gender of the patients $(p>0.05)$.

Based on mean scores, the highest GSTP1 expression was detected in pituitary adenomas, followed by meningiomas and metastases. The highest GSTM1 expression was detected in patients with pituitary adenomas. The highest CYP1A1 expression was detected in pituitary adenomas and the highest CYP1B1 expression was in meningiomas (Table 2).

There was no GSTP1 expression (score $=0$ ) in 26 normal tissues, while this score was observed in 12 tumor tissues. Mild GSTP1 expression was observed in 22 tumor samples and moderate expression in 16, and strong expression in 2 ( 1 pituitary adenoma and 1 renal cell carcinoma). GSTP1 expression was significantly high in the tumor samples when compared with the normal-tissue samples. There was no significant difference between normal- and tumor-tissue samples based on GSTM1 expression.

There was significantly increased CYP1A1 and CYP1B1 expression in tumor tissues when compared with normal tissues. Severe CYP1A1 expression was observed in 5 tumor samples: meningioma (grade I) in 4 cases and pituitary adenoma in 1 case. All these patients were nonsmokers. One of them was male and the rest female.

The mean age was 50.3 years and the mean follow-up period in patients with meningioma was 7 months (4-12 months). Eleven of 12 patients were female and the other was male. There was a significant female predominance among meningioma patients. In addition, there was no mortality in this group.

The mean age was 58.2 years and the mean follow-up period was 7.1 months in patients with metastases (2-11 months). Seven of them were male and 5 were female. The most common origin for metastasis was lung in 5 patients, and the most common histological type was ade- 
nocarcinoma in 4 cases. One patient with lung adenocarcinoma metastasis died 2 months after the surgery for right frontal tumor.

The mean age was 46.8 years and the mean follow-up period was 6.9 months (4-10 months) in patients with GBM. One patient with left thalamic GBM died 8 months after the surgery despite radiotherapy and chemotherapy.

Five patients had pituitary adenoma. Three of them were female and 2 were male. Their mean age was 46.2 years and mean follow-up period 6.6 months (4-11 months). FSH expression was positive in 3 patients, $\mathrm{LH}$ expression in 2, and PRL, GH, and ACTH expressions in 1 patient each. All of them had a low Ki-67 proliferation index. Mean GSTP1 and CYP1A1 expression scores were high in this group when compared with metastases, GBM, and meningioma.

Considering the age of the patients, the youngest was the pituitary adenoma group and the oldest was the metastasis group.

\section{Discussion}

Over an 8-month period, 55 patients were operated on for 57 intracranial lesions. Fifty-four were tumors and 3 were radiation necrosis. The most common tumors were metastasis and meningioma. GST and CYP expressions were analyzed in the tumor samples. GSTP1 and CYPs expression was increased in the intracranial tumors. Pituitary adenomas had higher GSTP1 and CYP1A1 expression. GSTM1 expression was not different in the normal tissue and tumor tissue.

GST isoenzymes are everywhere in nature. They can be found in all organisms. This enzyme family is mostly involved in protection against inflammation and genetic alterations as a result of oxidative stress [18]. Most of these enzymes catalyze the conjugation of reduced glutathion with compounds that contain an electrophilic center through the formation of a thioether bond between the sulfur atom of glutathion and the substrate [12].

The cytosolic GSTP1 catalyzes the nucleophilic attack of reduced glutathione on the electrophilic center of toxic, xenobiotic, and chemotherapeutic compounds, leading to their S-conjugation and elimination [12]. A lack of GSTP1 expression increases susceptibility to cancer $[6,7$, 11, 13]. GSTP1 has been associated with drug resistance and therapy failure. Abnormal GSTP1 activity and expression as well as GSTP1 DNA hypermethylation have been identified in prostate, breast, liver, renal, and endometrial carcinomas [19].
An intracranial tumor develops when the cancer cells grow rapidly and become an abnormal mass lesion. Once the tumor has grown to a point that it has areas of hypoxia, the tumor tissue begins to secrete high amounts of vascular endothelial growth factor for the development of a new blood supply to the tumor. This vasculature is known as the blood-tumor barrier (BTB); it limits the movement of chemotherapeutic drugs into the tumor [20]. In our study, we did not observe the expression of GST or CYP expression in the BTB of intracranial tumors. It is therefore not possible to speculate that these enzymes may participate in decreasing the entry of chemotherapeutic agents into the tumor tissue.

Glial tumors and metastases are the most common intracranial tumors and have a poor prognosis despite surgery and adjuvant therapies [5]. GBM is one of the most lethal and heterogeneous human cancers $[4,5]$. It has a poor response to chemotherapy and radiotherapy. In the last 2 decades, many studies of brain tumors have reported that an association exists between susceptibility and the presence of GST variants in adult patients [11, 19, 21, 22]. In contrast, Lai et al. [23] conducted a meta-analysis of the association between the genetic polymorphisms of GSTs and the risk of adult brain tumors, and their results did not suggest any relationship between the two.

Hara et al. [24] investigated the expression of GSTP1 in 26 meningioma patients by immunohistochemical methods, and they found that tissues of meningotheliomatous meningiomas were always positive for the expression of GSTP1. Transitional meningiomas also showed this expression in their meningotheliomatous components. No staining reaction of GSTP1 was recognized in fibroblastic meningiomas, except for 2 cases with a tendency to meningotheliomatous differentiation. In our series, 12 patients had meningioma and 5 of these were meningotheliomatous, 3 were transitional, and 4 were mixed-type. Strong-to-moderate GSTP1 expression was observed in the mixed-type meningiomas. Mild GSTP1 expression was observed in the menintotheliomatous and transitional meningiomas. GSTM1 expression, however, was very weak in the meningiomas. Moderate CYP1A1 expression was detected in meningotheliomatous meningiomas. Mild-moderate CYP1B1 expression was seen in meningotheliomatous and transistional meningiomas. Our series thus showed a similar pattern to the series in Hara et al. [24], i.e., it was based on GSTP1 expression.

Grant and Ironside [25] investigated the expression of GSTs $(\pi, \alpha$, and $\mu)$ and CYPs in 30 consecutive glioma cases. They found that GST immunostaining was evident in astrocytes and endothelium but not in neurons or oli-
Kural/Kaya Kocdogan/Şimşek/ Oğuztüzün/Kaygın/Yılmaz/Bayram/Izci 
godendrocytes in the normal brain. They stated that GST $\pi$ was always the predominant subclass, although GST $\alpha$ and GST $\mu$ were also expressed in some tumors. In our series, there were 20 cases with glial tumors and most of these were GBMs $(n=9)$. CYP expression was more evident than GST expression in our GBM cases.

Molina-Ortiz et al. [26] analyzed the gene expression patterns of CYP1A1, CYP1A2, CYP1B1, CYP2E1, CYP2W1, CYP3A4, and CYP3A5 in tumor and adjacent nontumor tissues from 13 pediatric rhabdomyosarcoma patients using real-time quantitative RT-PCR. In addition, they determined protein concentration of CYPs by Western blot and compared expression levels with the clinical and pathological data of the patients. They concluded that CYP1A1 and CYP1A2 expression is negligible and that the overexpression of CYP2W1, CYP3A4, and CYP$3 \mathrm{~A} 5$ in tumor tissues may be involved in rhabdomyosarcoma chemoresistance. Their study is important because it showed upregulation of mRNA expression levels of the members of CYP3 family in rhabdomyosarcoma samples. The results of our study are quite different. We analyzed intracranial tumors and used immunohistochemical methods to analyze the expression of CYP1A1 and CYP1B1. Interestingly, we found that CYP1A1 expression was increased in pituitary adenomas while CYP1B1 expression was decreased. This is different from the other tumor types. Although the aryl-hydrocarbon receptor signaling pathway regulates the production of CYP1B1 and CYP$1 \mathrm{~A} 1$, different expressions of these enzymes in pituitary adenomas may depend on cell-specific factors in cells of these tumors. This different expression was previously reported in breast cancers [27].

Hara et al. [28] also examined expression of GSTP1 in 31 gliomas and 6 normal brain-tissue specimens, and showed that benign astrocytomas had diffuse, weak GSTP1 immunostaining, resembling that of normal glial cells. With increasing grade, the gliomas showed a strongly positive reaction for GSTP1 but normal glial cells showed only a weak immunostaining response for GSTP1 in the cytoplasm or some nuclear membranes. In the glioma patients, the tumors showed a strongly positive reaction for GSTP1 with increasing grade. In our series, 20 patients had glial tumors, 9 of which were GBMs. There was mild expression of GSTP1 in the GBM samples; this expression was higher than in normal tissue.

Juillerat-Jeanneret et al. [11] analyzed the tumor specimens of 14 patients with a diagnosis of GBM and evaluated the expression of MGMT and GST. They also tried to ascertain whether the expression of GSTP1 and/or MGMT may be predictive of the response of human GBM

Glutathione-S-transferase and

Cytochrome p450 in Brain Tumors cells to alkylating agents and if this response may be improved by combining an alkylating agent with a GST or MGMT inhibitor. They found that the heterogeneity of the expression of MGMT and GSTP1 in human GBMs and GBM cell lines is an intrinsic property of these cells, rendering a variable but relevant fraction of these tumors unresponsive to alkylating agents.

Stavrinou et al. [14] investigated gene and protein expression in a cohort of 77 brain tumors using qRT-PCR and Western blot and showed that the various tumors of the central nervous system show different patterns of drug-metabolizing enzymes. In addition, meningiomas exhibited significantly higher expression levels. This expression was shown on both a transcriptional and translational level for GSTP1 and GSTM1. In our series, there were fewer patients $(n=55)$ than in their study $(n=77)$, but we found similar results, as meningiomas showed higher GSTP1, CYP1A1, and CYP1B1 expression than GBMs and metastases. Only pituitary adenomas showed more expression than meningiomas, which have a very different origin and behavior from meningiomas and gliomas.

There are also studies on the relationship between GSTP1, GSTM1, and pituitary tumors [29-31]. Yuan et al. [31] evaluated the GSTP1 expression level and GSTP1 DNA methylation status in 53 pituitary adenomas and found that GSTP1 inactivation through CpG hypermethylation is common in pituitary adenomas and may contribute to aggressive pituitary tumor behavior. Theirs was quite a large series; in our series, pituitary adenoma was diagnosed in 5 patients. There was a moderate-to-strong GSTP1 expression in pituitary adenomas and none of them showed aggressive behavior. Although our series was composed of a very low number of patients with pituitary adenomas, it is important to state that these tumors have strong GSTP1 activity when compared with meningiomas and glial tumors.

The limitations of our study were the low number of patients, the different histological types, and the lack of a comparison with responses to chemotherapy.

\section{Conclusion}

GSTP1 and CYP expressions are increased in intracranial tumors. Pituitary adenomas have strong expression of GSTP1 and CYP1A1, but this is statistically not significant. This strong GSTP1 expression can make medical treatment difficult. Our results need to be confirmed with larger series and different types of metabolizing enzymes. 


\section{Acknowledgments}

This study was partly supported by the Turkish Neurosurgical Society. We are grateful to Dr. Gulsah Kose for the statistical analysis.

\section{Statement of Ethics}

This study received the approval of the Kecioren Research and Education Hospital Ethics Committee.

\section{References}

1 Patchell RA. Overview of intracranial tumors. In: MSD Manual, Professional Version 2012. Available from: www.msdmanuals.com/professional/neurologic-disorders/intracranialand-spinal-tumors/overview-of-intracranialtumors.

2 Bogdanovic L, Savic S, Basta-Jovanovic G, Radojevic-Skodric S, Bogdanovic J. Death caused by undiagnosed primary intracranial neoplasmas - an autopsy study. Rev Med Leg. 2011;19(2):107-10.

3 Drewes C, Sagberg LM, Jakola AS, Gulati S, Solheim O. Morbidity after intracranial tumor surgery: sensitivity and specificity of retrospective review of medical records compared with patient-reported outcomes at 30 days. J Neurosurg. 2015 Oct;123(4):972-7.

4 Park IK, Hu K, Lee KW, et al. Clinical review of 30 cases of intracranial tumors. J Korean Neurosurg Soc. 1972;1:45-50.

5 Izci Y. Biomarkers for brain gliomas. In: Barh D, Carpi A, Verma M, Gunduz M, editors. Cancer Biomarkers Minimal and Noninvasive Early Diagnosis and Prognosis. Boca Raton: CRC Press, Taylor and Francis Group; 2014. pp. 199-218.

6 Backos DS, Franklin CC, Reigan P. The role of glutathione in brain tumor drug resistance. Biochem Pharmacol. 2012 Apr;83(8):100512.

7 Lo HW, Ali-Osman F. Genetic polymorphism and function of glutathione S-transferases in tumor drug resistance. Curr Opin Pharmacol. 2007 Aug;7(4):367-74.

8 Agundez JA. Cytochrome P450 gene polymorphism and cancer. Curr Drug Metab. 2004 Jun;5(3):211-24.

9 Izci Y, Gürkanlar D, Timurkaynak E. Multicentric gliomas: still remains a controversial issue. Turk Neurosurg. 2005;15:71-5.

10 Akay KM, Baysefer A, Kayali H, et al. Glioblastoma multiforme: the comparison of radiological findings, surgery and prognosis. Gulhane Tip Derg. 2002;44:142-8.

11 Juillerat-Jeanneret L, Bernasconi CC, Bricod C, Gros S, Trepey S, Benhattar J, et al. Heterogeneity of human glioblastoma: glutathioneS-transferase and methylguanine-methyltransferase. Cancer Invest. $2008 \mathrm{Jul} ; 26(6)$ : 597-609.

12 Nebert DW, Vasiliou V. Analysis of the glutathione S-transferase (GST) gene family. Hum Genomics. 2004 Nov; 1(6):460-4.
13 Sherratt PJ, Hayes JD. Glutathion-S-Transferases. In: Ionnides C, editor. Enzyme Systems that Metabolise Drugs and other Xenobiotics. West Sussex, UK: John Wiley\&Sons Ltd; 2002. pp. 319-52.

14 Stavrinou P, Mavrogiorgou MC, Polyzoidis K, Kreft-Kerekes V, Timmer M, Marselos M, et al. Expression profile of genes related to drug metabolism in human brain tumors. PLoS One. 2015 Nov; 10(11):e0143285.

15 Androutsopoulos VP, Tsatsakis AM, Spandidos DA. Cytochrome P450 CYP1A1: wider roles in cancer progression and prevention. BMC Cancer. 2009 Jun;9(1):187.

16 Hanna IH, Dawling S, Roodi N, Guengerich FP, Parl FF. Cytochrome P450 1B1 (CYP1B1) pharmacogenetics: association of polymorphisms with functional differences in estrogen hydroxylation activity. Cancer Res. 2000 Jul;60(13):3440-4.

17 Hashibe M, Brennan P, Strange RC, Bhisey R, Cascorbi I, Lazarus $\mathrm{P}$, et al. Meta- and pooled analyses of GSTM1, GSTT1, GSTP1, and CYP1A1 genotypes and risk of head and neck cancer. Cancer Epidemiol Biomarkers Prev. 2003 Dec;12(12):1509-17.

18 Solak B, Karkucak M, Turan H, Ocakoğlu G, Özemri Sağ Ş, Uslu E, et al. Glutathione Stransferase $\mathrm{M} 1$ and $\mathrm{T} 1$ gene polymorphisms in patients with chronic plaque-type psoriasis: A case-control study. Med Princ Pract. 2016;25(2):155-8.

19 Parl FF. Glutathione S-transferase genotypes and cancer risk. Cancer Lett. 2005 Apr;221(2): 123-9.

20 van Tellingen $\mathrm{O}$, Yetkin-Arik B, de Gooijer MC, Wesseling P, Wurdinger T, de Vries HE. Overcoming the blood-brain tumor barrier for effective glioblastoma treatment. Drug Resist Updat. 2015 Mar; 19:1-12.

21 Geng P, Li J, Wang N, Ou J, Xie G, Sa R, et al. Genetic contribution of polymorphisms in glutathione S-transferases to brain tumor risk. Mol Neurobiol. 2016 Apr;53(3):173040.

22 Pinarbasi H, Silig Y, Gurelik M. Genetic polymorphisms of GSTs and their association with primary brain tumor incidence. Cancer Genet Cytogenet. 2005 Jan;156(2):144-9.
23 Lai R, Crevier L, Thabane L. Genetic polymorphisms of glutathione S-transferases and the risk of adult brain tumors: a meta-analysis. Cancer Epidemiol Biomarkers Prev. 2005 Jul;14(7):1784-90

24 Hara A, Yamada H, Sakai N, Hirayama H, Tanaka T, Mori H. Immunohistochemical expression of glutathione S-transferase placental type (GST-pi), a detoxifying enzyme, in normal arachnoid villi and meningiomas. Virchows Arch A Pathol Anat Histopathol. 1990;417(6):493-6.

25 Grant R, Ironside JW. Glutathione S-transferases and cytochrome P450 detoxifying enzyme distribution in human cerebral glioma. J Neurooncol. 1995;25(1):1-7.

26 Molina-Ortiz D, Camacho-Carranza R, González-Zamora JF, Shalkow-Kalincovstein J, Cárdenas-Cardós R, Ností-Palacios R, et al. Differential expression of cytochrome P450 enzymes in normal and tumor tissues from childhood rhabdomyosarcoma. PLoS One. 2014 Apr;9(4):e93261.

27 Spink DC, Spink BC, Cao JQ, DePasquale JA, Pentecost BT, Fasco MJ, et al. Differential expression of CYP1A1 and CYP1B1 in human breast epithelial cells and breast tumor cells. Carcinogenesis. 1998 Feb;19(2):291-8.

28 Hara A, Yamada H, Sakai N, Hirayama H, Tanaka T, Mori H. Immunohistochemical demonstration of the placental form of glutathione S-transferase, a detoxifying enzyme in human gliomas. Cancer. 1990 Dec;66(12): 2563-8.

29 Perrett CW, Clayton RN, Pistorello M, Boscaro M, Scanarini M, Bates AS, et al. GSTM1 and CYP2D6 genotype frequencies in patients with pituitary tumours: effects on P53, ras and gsp. Carcinogenesis. $1995 \mathrm{Jul}$;6(7): 1643-5.

30 Fryer AA, Zhao L, Alldersea J, Boggild MD, Perrett CW, Clayton RN, et al. The glutathione S-transferases: polymerase chain reaction studies on the frequency of the GSTM1 0 genotype in patients with pituitary adenomas. Carcinogenesis. 1993 Apr;14(4):563-6.

31 Yuan Y, Qian ZR, Sano T, Asa SL, Yamada S, Kagawa N, et al. Reduction of GSTP1 expression by DNA methylation correlates with clinicopathological features in pituitary adenomas. Mod Pathol. 2008 Jul;21(7):856-65. 